Gut and Liver, Vol. 12, No. 2, March 2018, pp. 190-200

\title{
Combination of Transient Elastography and an Enhanced Liver Fibrosis Test to Assess the Degree of Liver Fibrosis in Patients with Chronic Hepatitis B
}

\author{
Ja Yoon $\mathrm{Heo}^{1}$, Beom Kyung Kim ${ }^{1,2}$, Jun Yong Park ${ }^{1,2}$, Do Young Kim ${ }^{1,2}$, Sang Hoon Ahn ${ }^{1,2}$, Hyon-Suk Kim ${ }^{3}$, Young Nyun \\ Park ${ }^{4}$, Kwang-Hyub Han ${ }^{1,2}$, Kijun Song ${ }^{5}$, and Seung Up Kim ${ }^{1,2}$ \\ ${ }^{1}$ Department of Internal Medicine, ${ }^{2}$ Institute of Gastroenterology, Departments of ${ }^{3}$ Laboratory Medicine, ${ }^{4}$ Pathology, and ${ }^{5}$ Biostatics, Yonsei \\ University College of Medicine, Seoul, Korea
}

Background/Aims: Liver stiffness (LS) was assessed using transient elastography, and the enhanced liver fibrosis (ELF) test was performed to accurately assess fibrotic burden. We validated the LS-ELF algorithm and investigated whether the sequential LS-ELF algorithm performs better than concurrent combination of these analyses in chronic hepatitis $\mathrm{B}(\mathrm{CHB})$ patients. Methods: Between 2009 and 2013, 222 CHB patients who underwent liver biopsy (LB), as well as LS measurement and the ELF test, were enrolled. Results: Advanced fibrosis ( $\mathrm{F} 3$ ) and cirrhosis (F4) were identified in 141 (63.6\%) and 118 (53.2\%) patients, respectively. Areas under receiver operating characteristic curve for LS predictions of $\geq \mathrm{F} 3$ ( 0.887 vs 0.703 ) and $F 4$ ( 0.853 vs 0.706 ) were significantly higher than the ELF test (all $\mathrm{p}<0.001$ ). Based on the LS-ELF algorithm, $60.4 \%$ to $71.6 \%$ and $55.7 \%$ to $66.3 \%$ of patients could have avoided LB to exclude $\geq F 3$ and F4, respectively, whereas $68.0 \%$ to $78.7 \%$ and $63.5 \%$ to $66.1 \%$ of patients could have avoided LB to confirm $\geq F 3$ and $F 4$, respectively. When confirmation and exclusion strategies were applied simultaneously, $69.4 \%$ to $72.5 \%$ and $60.8 \%$ to $65.3 \%$ of patients could have avoided LB and been diagnosed as $\geq F 3$ and F4, respectively. The proportion of patients who correctly avoided LB for the prediction of $\geq \mathrm{F} 3$ (69.4\% to $72.5 \%$ vs $42.3 \%$ to $59.0 \%)$ and $F 4(60.8 \%$ to $65.3 \%$ vs $23.9 \%$ to 49.5\%) based on the sequential LS-ELF algorithm was significantly higher than the concurrent combination (all $p<0.05$ ). Conclusions: The sequential LS-ELF algorithm conferred a greater probability of avoiding LB in CHB patients to diagnose advanced fibrosis and cirrhosis, and this test performed significantly better than the concurrent combination. (Gut Liver 2018;12:190-200)
Key Words: Hepatitis B, chronic; Enhanced liver fibrosis; Liver cirrhosis; Transient elastography; Liver stiffness

\section{INTRODUCTION}

Chronic hepatitis B (CHB) includes a diverse spectrum of diseases, ranging from asymptomatic infection to severely fulminant hepatitis and hepatic decompensation. ${ }^{1,2}$ The extent of liver fibrosis is one of the main prognostic factors in $\mathrm{CHB}$, which is correlated with the risk of developing cirrhosis and liver-related complications. ${ }^{3}$ Fortunately, recently developed potent antiviral agents for $\mathrm{CHB}$ can prevent disease progression, reduce the risk of emerging hepatocellular carcinoma (HCC), and even reverse hepatitis B virus (HBV)-induced liver fibrosis. However, because the remaining fibrotic burden cannot be completely resolved in most CHB patients, they are still at higher risks of developing liver-related complcations, such as HCC. ${ }^{3}$ Thus, the degree of liver fibrosis must be evaluated in any assessment of the risk of HBV-related HCC development and determination of longterm prognosis, even in this era of potent and active antiviral therapy. ${ }^{4,5}$

Liver biopsy (LB) is considered a reference method for evaluating the extent of liver fibrosis; ${ }^{6}$ however, it has some issues including sampling error and inter-/intra-observer interpretational variability. ${ }^{7,8}$ In addition, LB is associated with a low risk of life-threatening procedure-related complications such as bleeding, perforation, and even death. ${ }^{9}$ Thus, various biochemical surrogates for LB, such as the FibroTest (FT), enhanced liver fibrosis (ELF) test, and Wisteria floribunda agglutinin-positive human Mac-2 binding protein, ${ }^{10,11}$ as well as physical tools such as transient elastography (TE) and acoustic radiation force impulse elastography, have been proposed to noninvasively assess

Correspondence to: Seung Up Kim ${ }^{\mathrm{a}}$ and Kijun Song ${ }^{\mathrm{b}}$

Departments of ${ }^{\mathrm{a}}$ Internal Medicine and ${ }^{\mathrm{b}}$ Biostatics, Yonsei University College of Medicine, 50-1 Yonsei-ro, Seodaemun-gu, Seoul 03722, Korea

${ }^{a}$ Tel: +82-2-2228-1982, Fax: +82-2-362-6884, E-mail: ksukorea@yuhs.ac

'Tel: +82-2-2228-2491, Fax: +82-2-2228-2491, E-mail: BIOSTAT@yuhs.ac

Received on February 23, 2017. Revised on May 12, 2017. Accepted on May 22, 2017. Published online October 27,2017

pISSN 1976-2283 eISSN 2005-1212 https://doi.org/10.5009/gnl17092

(a) This is an Open Access article distributed under the terms of the Creative Commons Attribution Non-Commercial License (http://creativecommons.org/licenses/by-nc/4.0) which permits unrestricted non-commercial use, distribution, and reproduction in any medium, provided the original work is properly cited. 
the fibrotic burden in patients with chronic liver diseases. ${ }^{12,13}$

Of these noninvasive surrogates, the ELF test incorporates three serologic markers: hyaluronic acid (HA), N-terminal propeptide of collagen type III (PIIINP), and tissue inhibitor of metalloproteinase-1 (TIMP-1). ${ }^{14}$ The ELF test has demonstrated high reproducibility, automaticity, and performance in predicting the extent of liver fibrosis in patients with chronic liver diseases of varying etiologies. ${ }^{15-18}$ In addition, several longitudinal studies have reported a significant association between the ELF test and liver-related events, HCC, and liver-related deaths in patients with chronic liver diseases. Similarly, liver stiffness (LS) assessed using TE exhibits high accuracy and reproducibility in diagnosing the degree of liver fibrosis. ${ }^{19}$ Several longitudinal studies have demonstrated the ability of TE to predict long-term prognosis and assess the risk of HCC and liver-related event development. $^{20-22}$

Based on the accuracy of LS and ELF, Wong et al..$^{23}$ recently proposed a sequential LS-ELF algorithm to diagnose advanced fibrosis and cirrhosis in CHB patients. An exclusion and confirmation strategy based on this algorithm could improve the diagnostic accuracy of the LS value or ELF test alone, allowing approximately $60 \%$ of patients to avoid LB. However, this algorithm has not been validated or compared to the concurrent use of LS and ELF. In addition, the performance of ELF in the study by Wong et al. $^{23}$ was relatively lower than that previously reported (area under receiver operating characteristic curve [AUC], 0.69 vs 0.86 to 0.92$).{ }^{15-18}$

Thus, in this single-center, retrospective cohort study, we investigated the diagnostic performance of LS assessed using TE and the ELF test for advanced fibrosis and cirrhosis, validated the LS-ELF algorithm, and determined whether sequential LSELF algorithm is superior to their concurrent combination in terms of preventing unnecessary LB in CHB patients.

\section{MATERIALS AND METHODS}

\section{Patients}

From 2009 to 2013, 265 CHB patients who underwent TE and LB at Severance Hospital, Yonsei University College of Medicine (Seoul, Korea) were considered eligible for inclusion in this study. The study population included $99 \mathrm{CHB}$ patients who were recruited in our previous study between 2009 and 2010. ${ }^{14} \mathrm{CHB}$ was defined as the persistent presence of serum HBV surface antigen for more than 6 months and HBV DNA positivity by the polymerase chain reaction assay. Before starting antiviral therapy, LB was performed to assess the severity of liver fibrosis. With informed consent, serum samples taken at the time of LB were stored in the Yonsei Liver Blood Bank system (approval number: 4-2009-0725).

The exclusion criteria were as follows: (1) measurement failure of LS value (valid shot=0); (2) unreliable LS value; (3) measurement failure of the ELF test; (4) a previous history of antivi- ral therapy and hepatic decompensation; (5) HCC at the time of LB or a past history of it; ${ }^{24}$ (6) liver specimen less than $15 \mathrm{~mm}$ in length; (7) alanine aminotransferase (ALT) level $>5 \times$ the upper limit of normal (ULN); (8) co-infection with human immunodeficiency virus or hepatitis $\mathrm{C}$ virus; (9) alcohol ingestion in excess of $40 \mathrm{~g} /$ day for more than 5 years; ${ }^{25}$ and (10) heart failure or pregnancy (Supplementary Fig. 1).

This study was performed in accordance with the ethical guidelines of the 1975 Declaration of Helsinki and was approved by the Institutional Review Board of Severance Hospital. Given its retrospective nature, written informed consent to access clinical data was not required.

\section{Histologic evaluation}

LB specimens were fixed in formalin and embedded in paraffin. Then standard hematoxylin and eosin and trichrome (Masson) stains were performed using $4-\mu \mathrm{m}$ sections. All of the liver tissue samples were evaluated by an experienced pathologist blinded to the patient clinical data, including the results of the TE and ELF tests. Liver histology was scored semi-quantitatively according to the Batts and Ludwig system. ${ }^{26}$ Fibrosis was staged (0 to 4) as follows: F0, no fibrosis; F1, portal fibrosis without septa; F2, portal fibrosis and few septa; F3, numerous septa without cirrhosis; and F4, cirrhosis.

\section{ELF measurement}

ELF was tested using serum samples at stored the time of LB. HA, PIIINP, and TIMP-1 levels were estimated using an ADVIA Centaur XP automated immunoanalyzer (Siemens Healthcare Diagnostics, Tarrytown, NY, USA). ELF was calculated by the following algorithm provided by the manufacturer: $\mathrm{ELF}=2.278+0.851 \ln (\mathrm{HA})+0.751 \ln (\mathrm{PIIINP})+0.394 \ln (\mathrm{TIMP}-1)$.

\section{LS measurement using TE}

At the time of enrollment, TE was performed by a well-trained technician (>50,000 examinations). Details of the technique and examination procedure were previously reported. ${ }^{19,27-29}$ LS values were expressed as kilopascals $(\mathrm{kPa})$. The interquartile range (IQR) was defined as an index of intrinsic variability of LS values, corresponding to the interval of LS measurement results containing 50\% of the valid measurements between the 25th and 75 th percentiles. In this study, only LS values with 10 validated measurements and a 60\% success rate (at minimum, both) were considered reliable. The median of successfully measured values was presumed representative of LS in a given patient only at IQR-to-median-value ratios $<0.3$.

\section{Sequential LS-ELF algorithm}

Wong et al..$^{23}$ proposed a sequential LS-ELF algorithm, in which confirmation and exclusion by the LS value were performed first. When the value was indeterminate, confirmation and exclusion by the ELF test were performed in sequence, and 
when both values were nondiagnostic, LB was considered. A confirmatory strategy was defined as accurate if the posttest probability was $>90 \%$, and the exclusion strategy was defined as accurate if the posttest probability was $<10 \%$. The LS and ELF value cutoffs proposed by Wong et al. ${ }^{23}$ were adopted as external cutoff values in this study. The external LS value cutoffs were as follows: $\geq \mathrm{F} 3,6.0 \mathrm{kPa}$ and $\mathrm{F} 4,7.5 \mathrm{kPa}$ for at least 90\% sensitivity; $\geq \mathrm{F} 3,10.2 \mathrm{kPa}$ and F4, $12.0 \mathrm{kPa}$ for at least $90 \%$ specificity; and $\geq \mathrm{F} 3,9.0 \mathrm{kPa}$ and $\mathrm{F} 4,10.0 \mathrm{kPa}$ for the maximum sum of sensitivity and specificity. Those for the ELF test were as follows: $\geq F 3$, 8.4 and F4, 8.8 for at least 90\% sensitivity; $\geq F$ 3, 10.8 and F4, 11.1 for at least 90\% specificity; and $\geq F 3,9.8$ and F4, 9.5 for the maximum sum of sensitivity and specificity.

\section{Concurrent LS-ELF algorithm}

The accuracy of the concurrent use of LS and ELF test values was compared to the sequential LS-ELF algorithm by Wong et al.'s method. ${ }^{23}$ To establish the strategy for the concurrent use of LS and ELF test values in our study cohort and comparing the accuracy of this concurrent LS-ELF algorithm with that of the sequential LS-ELF algorithm, we adopted two strategies that have been used in previous studies. First, Boursier et al. ${ }^{30}$ proposed the concurrent combination of FibroMeter and LS values. In this algorithm, a forward binary logistic regression was performed using both values. Using the regression score, the 90\% negative predictive value (NPV) and 90\% positive predictive value (PPV) were set. LB was considered required when the regression score was in the indeterminate zone between the two thresholds. Second, Castéra et al. ${ }^{10}$ proposed a different concurrent combination of FT and LS values, in which LB was considered when the FT and LS values did not match.

\section{ULN of ALT and stratification according to ALT level}

Because patients at the same fibrosis stage but with higher ALT levels tend to exhibit higher LS values, the diagnostic performances of LS, ELF, and the LS-ELF algorithm were calculated separately according to ALT level (ALT $\leq$ ULN and ALT 1-5x ULN). ${ }^{31}$ In this study, ULNs of 67 IU/L for males and 55 IU/L for females, as used by Wong et al., ${ }^{23}$ were adopted. In addition, we used another ULN of $40 \mathrm{IU} / \mathrm{L}\left(\mathrm{ULN}_{\text {Korea }}\right.$ ) for both sexes which has been used in Korea. ${ }^{14,20,27,28}$ Based on ULN $_{\text {Korea }}$, 218 patients showed an ALT level $\leq 5 \times$ ULN and were used for subgroup analyses.

\section{Statistical analysis}

The SPSS version 18.0 (SPSS Inc., Chicago, IL, USA) was utilized for all of the statistical analysis. Data are expressed as medians (IQR) or number (\%) as appropriate. Pearson correlation coefficient was used to test the correlations among LS and ELF values with the other variables. The performance of LS and ELF in terms of predicting the degree of liver fibrosis was evaluated by AUCs with 95\% confidence intervals (CIs).
The DeLong method was used to compare the AUC values of LS and ELF. The sensitivity, specificity, PPV, NPV, positive and negative likelihood ratios of LS, ELF, and the LS-ELF algorithm were calculated to evaluate the accuracy of the determinants. Internal cutoffs were calculated from our study population and external cutoffs were from Wong et al. ${ }^{23}$ Cutoff $_{\text {se }}$ was defined as the cutoff value which had at least 90\% sensitivity and cutoff $_{\text {sp }}$ was defined as the cutoff value which had at least $90 \%$

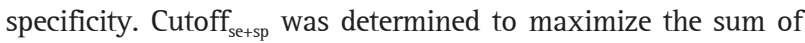
sensitivity and specificity from receiver operating characteristic curve analyses, and the corresponding diagnostic indices were calculated. The proportion of patients with correctly avoided biopsy was compared among the algorithms by the McNemar test. The cumulative incidence rate of HCC was calculated using the Kaplan-Meier method and compared using log-rank test. A value of $p<0.05$ was considered statistically significant.

\section{RESULTS}

\section{Patient characteristics}

A total of 265 consecutive CHB patients who underwent LB, LS measurement, and the ELF test were considered eligible for this study. Fifteen patients were excluded due to measurement

Table 1. Baseline Characteristics $(n=222)$

\begin{tabular}{|c|c|}
\hline Variable & Value \\
\hline \multicolumn{2}{|l|}{ Demographic } \\
\hline Age, yr & $48(37-55)$ \\
\hline Male sex & $144(64.9)$ \\
\hline Body mass index, $\mathrm{kg} / \mathrm{m}^{2}$ & $23.7(21.7-25.6)$ \\
\hline Diabetes mellitus & $14(6.3)$ \\
\hline Hypertension & $27(12.2)$ \\
\hline \multicolumn{2}{|l|}{ Laboratory } \\
\hline Alanine aminotransferase, IU/L & $42(30-64)$ \\
\hline Serum albumin, g/dL & $4.2(3.9-4.5)$ \\
\hline Total bilirubin, mg/dL & $0.7(0.6-0.9)$ \\
\hline$\gamma$-Glutamyl transpeptidase, IU/L & $37(25-69)$ \\
\hline Platelet count, $10^{9} / \mathrm{L}$ & $169(122-201)$ \\
\hline HBeAg positivity & $115(51.8)$ \\
\hline HBV DNA, copies/mL & $616,000(19,950-14,775,000)$ \\
\hline \multicolumn{2}{|l|}{ Histological } \\
\hline Length of biopsy samples, cm & $1.7(1.6-2.0)$ \\
\hline \multicolumn{2}{|l|}{ Fibrosis stage } \\
\hline $\mathrm{F} 1 / \mathrm{F} 2 / \mathrm{F} 3 / \mathrm{F} 4$ & 39 (17.6)/42 (18.9)/23 (10.4)/118 (53.1) \\
\hline \multicolumn{2}{|l|}{ Noninvasive fibrosis assessment } \\
\hline LS value, $\mathrm{kPa}$ & $10.2(6.9-15.9)$ \\
\hline ELF test & $9.7(8.8-10.4)$ \\
\hline
\end{tabular}

Data are presented as median (interquartile range) or number (\%). $\mathrm{HBeAg}$, hepatitis B e antigen; HBV, hepatitis B virus; LS, liver stiffness; ELF, enhanced liver fibrosis. 
A

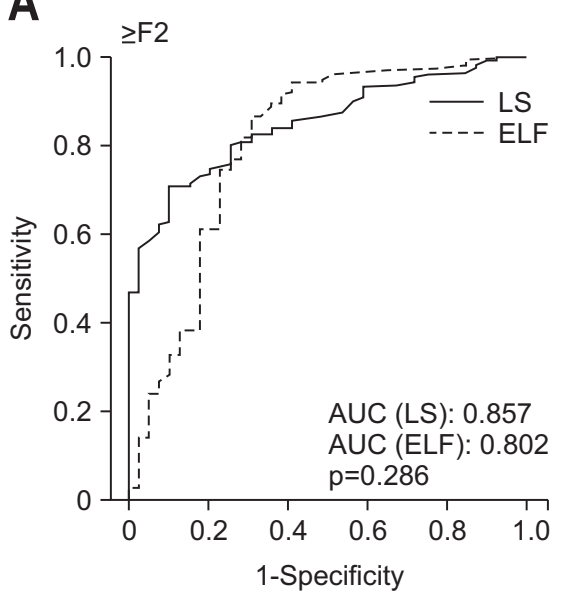

B

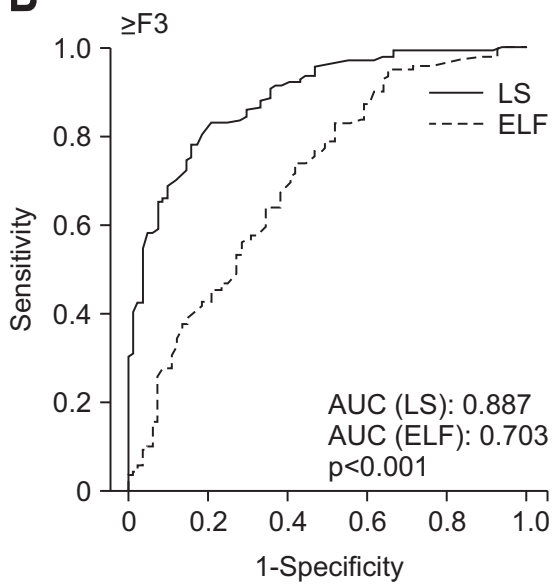

C

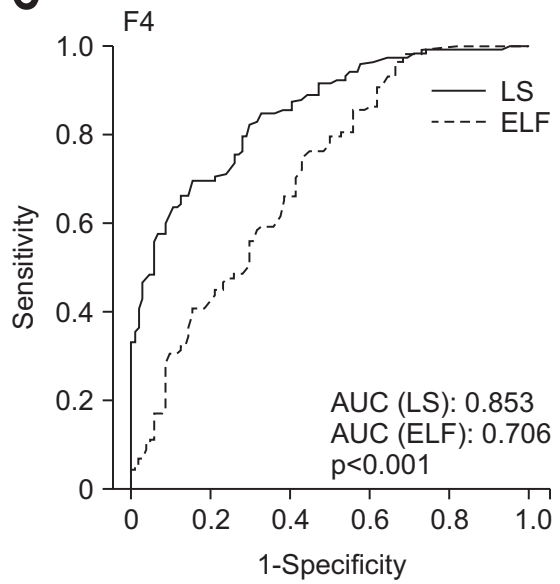

Fig. 1. Receiver operating characteristic curves of liver stiffness (LS) and enhanced liver fibrosis (ELF) values to predict fibrosis stages. LS and ELF values were similarly predictive of $\geq F 2$ stage (A) (area under receiver operating characteristic curve [AUC], 0.857 vs $0.802 ; p=0.286$ ), whereas the LS value was superior to the ELF value for predicting the $\geq F 3$ (B) (AUC, 0.887 vs $0.703 ; p<0.001$ ) and F4 stages (C) (AUC, 0.853 vs 0.706 ; $\mathrm{p}<0.001)$.

Table 2. Diagnostic Indices of LS and ELF Values to Assess $\geq F 3$ and F4 Stages according to External and Internal Cutoff Values

\begin{tabular}{|c|c|c|c|c|c|c|c|c|c|c|c|c|c|c|}
\hline \multirow{2}{*}{ Variable } & \multicolumn{7}{|c|}{$\geq \mathrm{F} 3(\mathrm{n}=141)$} & \multicolumn{7}{|c|}{$\mathrm{F} 4(\mathrm{n}=118)$} \\
\hline & Cutoff & Sn, $\%$ & $\mathrm{Sp}, \%$ & PPV, \% & NPV, \% & LR+ & LR- & Cutoff & Sn, $\%$ & $\mathrm{Sp}, \%$ & PPV, \% & NPV, \% & $\mathrm{LR+}$ & LR- \\
\hline \multicolumn{15}{|l|}{ LS value* } \\
\hline \multicolumn{15}{|c|}{ Based on external cutoff } \\
\hline \multicolumn{15}{|l|}{ All } \\
\hline Sn $>90 \%$ & 6.0 & 97.2 & 44.4 & 75.3 & 90.0 & 1.74 & 0.06 & 7.5 & 91.5 & 51.0 & 67.9 & 84.1 & 1.86 & 0.16 \\
\hline $\mathrm{Sp}>90 \%$ & 10.2 & 70.2 & 87.7 & 90.8 & 62.8 & 5.68 & 0.33 & 12.0 & 59.3 & 91.3 & 88.6 & 66.4 & 6.85 & 0.44 \\
\hline $\mathrm{Sn}+\mathrm{Sp}_{\max }$ & 9.0 & 80.1 & 81.5 & 88.3 & 70.2 & 4.32 & 0.24 & 10.0 & 75.4 & 74.0 & 76.7 & 72.6 & 2.90 & 0.33 \\
\hline \multicolumn{15}{|l|}{$\mathrm{ALT} \leq \mathrm{ULN}$} \\
\hline $\mathrm{Sn}>90 \%$ & 6.0 & 97.4 & 41.3 & 80.9 & 86.4 & 1.66 & 0.06 & 6.0 & 98.0 & 31.3 & 68.8 & 90.9 & 1.42 & 0.06 \\
\hline $\mathrm{Sp}>90 \%$ & 9.0 & 80.3 & 82.6 & 92.2 & 62.3 & 4.61 & 0.23 & 12.0 & 57.6 & 89.1 & 89.1 & 57.6 & 5.26 & 0.47 \\
\hline $\mathrm{Sn}+\mathrm{Sp}_{\max }$ & 9.0 & 80.3 & 82.6 & 92.2 & 62.3 & 4.61 & 0.23 & 10.0 & 74.7 & 71.9 & 80.4 & 64.8 & 2.65 & 0.35 \\
\hline \multicolumn{15}{|c|}{ ALT 1-5× ULN } \\
\hline Sn $>90 \%$ & 7.5 & 95.8 & 65.7 & 65.7 & 95.8 & 2.79 & 0.06 & 7.5 & 94.7 & 57.5 & 51.4 & 95.8 & 2.22 & 0.09 \\
\hline $\mathrm{Sp}>90 \%$ & 12.0 & 58.3 & 97.1 & 93.3 & 77.3 & 20.40 & 0.42 & 12.0 & 68.4 & 95.0 & 86.7 & 86.4 & 13.68 & 0.33 \\
\hline $\mathrm{Sn}+\mathrm{Sp}_{\max }$ & 11.0 & 75.0 & 94.3 & 90.0 & 84.6 & 13.12 & 0.26 & 11.0 & 78.9 & 87.5 & 75.0 & 89.7 & 6.31 & 0.24 \\
\hline \multicolumn{15}{|c|}{ Based on internal cutoff } \\
\hline \multicolumn{15}{|l|}{ All } \\
\hline Sn $>90 \%$ & 7.6 & 90.8 & 64.2 & 81.5 & 80.0 & 2.53 & 0.14 & 7.6 & 91.5 & 51.0 & 67.9 & 84.1 & 1.86 & 0.16 \\
\hline $\mathrm{Sp}>90 \%$ & 10.5 & 66.0 & 90.1 & 92.1 & 60.3 & 6.67 & 0.37 & 11.8 & 60.2 & 91.3 & 88.8 & 66.9 & 6.95 & 0.43 \\
\hline $\mathrm{Sn}+\mathrm{Sp}_{\max }$ & 9.0 & 80.1 & 81.5 & 88.3 & 70.2 & 4.32 & 0.24 & 11.0 & 66.1 & 85.6 & 83.9 & 69.0 & 4.58 & 0.39 \\
\hline \multicolumn{15}{|l|}{$\mathrm{ALT} \leq \mathrm{ULN}$} \\
\hline $\mathrm{Sn}>90 \%$ & 7.5 & 90.6 & 60.9 & 85.5 & 71.8 & 2.31 & 0.15 & 7.6 & 90.9 & 50.0 & 73.8 & 78.0 & 1.81 & 0.18 \\
\hline $\mathrm{Sp}>90 \%$ & 10.4 & 66.7 & 91.3 & 95.1 & 51.9 & 7.66 & 0.36 & 12.3 & 55.6 & 90.6 & 90.2 & 56.9 & 5.92 & 0.49 \\
\hline $\mathrm{Sn}+\mathrm{Sp}_{\max }$ & 9.0 & 80.3 & 82.6 & 92.2 & 62.3 & 4.61 & 0.23 & 9.0 & 82.8 & 68.8 & 80.4 & 72.1 & 2.65 & 0.24 \\
\hline \multicolumn{15}{|c|}{ ALT $1-5 \times$ ULN } \\
\hline Sn $>90 \%$ & 8.0 & 91.7 & 68.6 & 66.7 & 92.3 & 2.91 & 0.12 & 8.0 & 94.7 & 57.5 & 51.4 & 95.8 & 2.22 & 0.09 \\
\hline $\mathrm{Sp}>90 \%$ & 10.6 & 79.2 & 91.4 & 86.4 & 86.5 & 9.23 & 0.22 & 11.5 & 78.9 & 90.0 & 78.9 & 90.0 & 7.89 & 0.23 \\
\hline $\mathrm{Sn}+\mathrm{Sp}_{\max }$ & 10.6 & 79.2 & 91.4 & 86.4 & 86.5 & 9.23 & 0.22 & 11.5 & 78.9 & 90.0 & 78.9 & 90.0 & 7.89 & 0.23 \\
\hline
\end{tabular}


Table 2. Continued

\begin{tabular}{|c|c|c|c|c|c|c|c|c|c|c|c|c|c|c|}
\hline \multirow{2}{*}{ Variable } & \multicolumn{7}{|c|}{$\geq \mathrm{F} 3(\mathrm{n}=141)$} & \multicolumn{7}{|c|}{$\mathrm{F} 4(\mathrm{n}=118)$} \\
\hline & Cutoff & $\mathrm{Sn}, \%$ & $\mathrm{Sp}, \%$ & PPV, \% & NPV, \% & $\mathrm{LR}+$ & LR- & Cutoff & $\mathrm{Sn}, \%$ & $\mathrm{Sp}, \%$ & PPV, \% & NPV, \% & $\mathrm{LR}+$ & LR- \\
\hline \multicolumn{15}{|l|}{ ELF test } \\
\hline \multicolumn{15}{|c|}{ Based on external cutoff } \\
\hline \multicolumn{15}{|l|}{ All } \\
\hline $\mathrm{Sn}>90 \%$ & 8.4 & 95.0 & 34.6 & 71.7 & 80.0 & 1.42 & 0.14 & 8.8 & 86.4 & 38.5 & 61.4 & 71.4 & 1.40 & 0.35 \\
\hline $\mathrm{Sp}>90 \%$ & 10.8 & 24.8 & 92.6 & 85.4 & 41.4 & 3.35 & 0.81 & 11.1 & 21.2 & 91.3 & 73.5 & 50.5 & 2.44 & 0.86 \\
\hline $\mathrm{Sn}+\mathrm{Sp}_{\max }$ & 9.8 & 53.2 & 71.6 & 76.5 & 46.8 & 1.87 & 0.65 & 9.5 & 66.1 & 58.7 & 64.5 & 60.4 & 1.59 & 0.57 \\
\hline \multicolumn{15}{|l|}{$\mathrm{ALT} \leq \mathrm{ULN}$} \\
\hline $\mathrm{Sn}>90 \%$ & 8.4 & 94.9 & 30.4 & 77.6 & 70.0 & 1.36 & 0.18 & 8.8 & 85.9 & 39.1 & 68.5 & 64.1 & 1.40 & 0.36 \\
\hline $\mathrm{Sp}>90 \%$ & 10.8 & 23.9 & 91.3 & 87.5 & 32.1 & 2.75 & 0.83 & 11.1 & 22.2 & 92.2 & 81.5 & 43.4 & 2.84 & 0.84 \\
\hline $\mathrm{Sn}+\mathrm{Sp}_{\max }$ & 9.8 & 51.3 & 76.1 & 84.5 & 38.0 & 2.14 & 0.64 & 9.5 & 65.7 & 62.5 & 73.0 & 54.1 & 1.50 & 0.54 \\
\hline \multicolumn{15}{|c|}{ ALT 1-5x ULN } \\
\hline $\mathrm{Sn}>90 \%$ & 9.2 & 75.0 & 48.6 & 50.0 & 73.9 & 1.45 & 0.51 & 9.5 & 68.4 & 52.5 & 40.6 & 77.8 & 1.44 & 0.60 \\
\hline $\mathrm{Sp}>90 \%$ & 10.8 & 29.2 & 94.3 & 77.8 & 66.0 & 5.10 & 0.75 & 11.1 & 15.8 & 90.0 & 42.9 & 69.2 & 1.57 & 0.93 \\
\hline $\mathrm{Sn}+\mathrm{Sp}_{\max }$ & 9.8 & 62.5 & 65.7 & 55.6 & 71.9 & 1.82 & 0.57 & 9.5 & 68.4 & 52.5 & 40.6 & 77.8 & 1.44 & 0.60 \\
\hline \multicolumn{15}{|c|}{ Based on internal cutoff } \\
\hline \multicolumn{15}{|l|}{ All } \\
\hline $\mathrm{Sn}>90 \%$ & 8.6 & 90.8 & 35.8 & 71.1 & 69.0 & 1.41 & 0.25 & 8.7 & 90.7 & 37.5 & 62.2 & 78.0 & 1.45 & 0.24 \\
\hline $\mathrm{Sp}>90 \%$ & 10.8 & 24.8 & 92.6 & 85.4 & 41.4 & 3.35 & 0.81 & 10.7 & 28.8 & 91.3 & 79.1 & 53.1 & 3.32 & 0.77 \\
\hline $\mathrm{Sn}+\mathrm{Sp}_{\max }$ & 8.4 & 95.0 & 34.6 & 71.7 & 80.0 & 1.42 & 0.14 & 8.4 & 98.3 & 31.7 & 62.0 & 94.3 & 1.43 & 0.05 \\
\hline \multicolumn{15}{|l|}{$\mathrm{ALT} \leq \mathrm{ULN}$} \\
\hline $\mathrm{Sn}>90 \%$ & 8.6 & 90.6 & 32.6 & 77.4 & 57.7 & 1.34 & 0.28 & 8.7 & 90.9 & 37.5 & 69.2 & 72.7 & 1.38 & 0.35 \\
\hline $\mathrm{Sp}>90 \%$ & 10.7 & 25.6 & 91.3 & 88.2 & 32.6 & 2.94 & 0.81 & 10.6 & 31.3 & 90.6 & 83.8 & 46.0 & 3.34 & 0.75 \\
\hline $\mathrm{Sn}+\mathrm{Sp}_{\max }$ & 9.0 & 77.8 & 52.2 & 80.5 & 48.0 & 1.62 & 0.42 & 8.5 & 97.0 & 31.3 & 68.6 & 87.0 & 1.41 & 0.09 \\
\hline \multicolumn{15}{|c|}{ ALT $1-5 \times$ ULN } \\
\hline $\mathrm{Sn}>90 \%$ & 8.6 & 91.7 & 40.0 & 51.2 & 87.5 & 1.52 & 0.20 & 8.6 & 94.7 & 37.5 & 41.9 & 93.8 & 1.51 & 0.14 \\
\hline $\mathrm{Sp}>90 \%$ & 10.8 & 29.2 & 94.3 & 77.8 & 66.0 & 5.10 & 0.75 & 11.1 & 15.8 & 90.0 & 42.9 & 69.2 & 1.57 & 0.93 \\
\hline $\mathrm{Sn}+\mathrm{Sp}_{\max }$ & 8.4 & 95.8 & 40.0 & 52.3 & 93.3 & 1.59 & 0.10 & 8.4 & 100.0 & 37.5 & 43.2 & 100.0 & 1.60 & 0.00 \\
\hline
\end{tabular}

LS, liver stiffness; ELF, enhanced liver fibrosis; Sn, sensitivity; Sp, specificity; PPV, positive predictive value; NPV, negative predictive value; LR, likelihood ratio; ALT, alanine aminotransferase; ULN, upper limit of normal.

*LS values were expressed as kilopascals (kPa).

failure of LS value, unreliable LS value, or measurement failure of ELF test. Of the 250 patients with valid LS values and ELF test results, 28 were excluded according to the exclusion criteria. Finally, a total of 222 patients were selected for statistical analysis.

The baseline characteristics of the study population (144 males and 78 females) are shown in Table 1 . The median age and body mass index was 48 years and $23.7 \mathrm{~kg} / \mathrm{m}^{2}$, respectively. Histological fibrosis staging was as follows: F1 in 39, F2 in 42, F3 in 23, and F4 in 118 patients, respectively. The median LS and ELF values were $10.2 \mathrm{kPa}$ and 9.7, respectively.

\section{Correlation among LS and ELF values with the other variables}

Age, total bilirubin, $\gamma$-glutamyl transpeptidase, and fibrosis stage significantly increased with increasing LS and ELF values, whereas serum albumin and platelet count significantly decreased (all $\mathrm{p}<0.05$ ). LS and ELF values were significantly correlated $(r=0.500, p<0.001)$, whereas ALT values did not show significant correlations with LS or ELF values $(p=0.067$ and $\mathrm{p}=0.494$, respectively) (Supplementary Fig. 1).

\section{Diagnostic performance of LS and ELF values}

The AUCs of LS value to predict $\geq F 2, \geq F 3$, and F4 fibrosis stage were 0.857 (95\% CI, 0.804 to 0.900), 0.887 (95\% CI, 0.837 to 0.925 ), and 0.853 (95\% CI, 0.799 to 0.897 ), respectively; and those of ELF were 0.802 (95\% CI, 0.743 to 0.852), 0.703 (95\% CI, 0.638 to 0.762 ), and 0.706 (95\% CI, 0.642 to 0.765$)$, respectively (Fig. 1). The performance of the LS and ELF values to predict $\geq F 2$ was statistically similar $(p=0.286)$, whereas the LS 
value was significantly superior to the ELF value in predicting $\geq$ F3 and F4 (both $\mathrm{p}<0.001$ ).

Of the study population ( $\mathrm{n}=222), 163$ patients $(73.4 \%)$ had ALT levels $\leq \mathrm{ULN}$ and 59 patients (26.4\%) had ALT level of $1-5 \times$ ULN. If ULN $_{\text {Korea }}$ was used ( $\left.\mathrm{n}=218\right), 98$ patients $(45.0 \%)$ had ALT levels $\leq \mathrm{ULN}$ and 120 patients (55.0\%) had ALT levels of 1-5x ULN. When the patients were stratified according to their ALT level (ALT $\leq$ ULN vs ALT $1-5 \times$ ULN) and ULN criteria, LS value was significantly superior to the ELF value for predicting $\geq \mathrm{F} 3$ (AUC, 0.859 to 0.910 vs 0.697 to 0.714 ) and F4 (AUC, 0.838 to 0.886 vs 0.697 to 0.720 ) (all $\mathrm{p}<0.05$ ) (Supplementary Table 1).

\section{Cutoff values of LS and ELF for predicting $\geq F 3$ and F4}

Diagnostic indices of LS and ELF value for predicting $\geq F 3$ and F4 were calculated according to external cutoff values from Hong Kong ${ }^{23}$ and internal cutoff values from our cohort were calculated (Table 2). In addition, the LS and ELF value cutoffs according to ALT level (ALT $\leq$ ULN vs ALT $1-5 \times$ ULN) were determined (Table 2).

For prediction of $\geq F 3$, the internal cutoff $_{\text {se }}$, cutoff $f_{\text {sp }}$, and cutoff $_{\text {setsp }}$ values were 7.6, 10.5, and $9.0 \mathrm{kPa}$, respectively for LS; and those for ELF were 8.6, 10.8, and 8.4, respectively. An elevated ALT level (1-5x ULN) was associated with a slightly increased LS cutoff value compared to a normal ALT value $\left(\leq \mathrm{ULN}\right.$ ) (cutoff $_{\mathrm{se}} 7.5 \mathrm{kPa} \rightarrow 8.0 \mathrm{kPa}$, cutoff ${ }_{\mathrm{sp}} 10.4 \mathrm{kPa} \rightarrow 10.6 \mathrm{kPa}$, and cutoff sesp $9.0 \mathrm{kPa} \rightarrow 10.6 \mathrm{kPa}$ ), but was not associated with a change in ELF value (cutoff se $^{8.6} \rightarrow 8.6$, cutoff $_{\text {sp }} 10.7 \rightarrow 10.8$, and cutoff $_{\text {se+sp }} 9.0 \rightarrow 8.4$ ). For prediction of F4, an elevated ALT level had varying effects on LS and ELF cutoff values in predicting F4. Similar results were obtained when ULN $_{\text {Korea }}$ was used (Supplementary Table 2).

\section{Diagnostic performance of LS, ELF, and the LS-ELF algorithm}

The diagnostic performance of LS, ELF, and the LS-ELF algorithm to exclude and confirm $\geq \mathrm{F} 3$ and F4 is shown in Table 3 . For reference, published diagnostic indices of Wong's cohort are also described in Table 3. Using a LS-ELF exclusion strategybased algorithm for our cohort, 60.4\% $(n=49)$ and 55.7\% of patients $(n=58)$ could avoid LB to exclude $\geq F 3$ and F4 using external cutoffs, respectively; whereas $71.6 \%(n=58)$ and $66.3 \%$ $(n=69)$ of patients could avoid LB to exclude $\geq F 3$ and F4 using internal cutoffs, respectively. In addition, the proportions of patients who could avoid LB to confirm $\geq \mathrm{F} 3$ and F4 using exter-

Table 3. Diagnostic Performance of LS, ELF, and the LS-ELF Algorithms to Exclude and Confirm $\geq F 3$ and F4

\begin{tabular}{|c|c|c|c|c|}
\hline \multirow{2}{*}{ Variable } & \multicolumn{2}{|c|}{ ELF test } & \multicolumn{2}{|c|}{ LS value* } \\
\hline & Internal & External & Internal & External \\
\hline \multicolumn{5}{|l|}{ Exclusion strategy } \\
\hline \multicolumn{5}{|l|}{$\geq \mathrm{F} 3(\mathrm{n}=81)$} \\
\hline \multirow[t]{2}{*}{ Cutoff value } & 8.6 & 8.4 & $7.5(\mathrm{ALT} \leq \mathrm{ULN})$ & $6.0(\mathrm{ALT} \leq \mathrm{ULN})$ \\
\hline & & & 8.0 (ALT 1-5× ULN) & 7.5 (ALT 1-5× ULN) \\
\hline Sensitivity, \% & 91.0 & 95.0 & 90.8 & 97.2 \\
\hline Specificity, \% & 33.3 & 34.6 & 64.2 & 51.9 \\
\hline PPV, \% & 68.6 & 71.7 & 81.5 & 77.8 \\
\hline NPV, \% & 69.8 & 80.0 & 80.0 & 91.3 \\
\hline $\mathrm{LR}+$ & 1.4 & 1.4 & 2.5 & 2.0 \\
\hline LR- & 0.3 & 0.1 & 0.1 & 0.1 \\
\hline No. of biopsy correctly avoided (\%) & $29(35.8)$ & $28(34.0)$ & $52(64.2)$ & $42(51.8)$ \\
\hline No. of incorrect diagnosis (\%) & $13(16.0)$ & 7 (8.6) & $13(16.0)$ & $4(4.9)$ \\
\hline \multicolumn{5}{|l|}{$\mathrm{F} 4(\mathrm{n}=104)$} \\
\hline \multirow[t]{2}{*}{ Cutoff value } & 8.7 & 8.8 & $7.6(\mathrm{ALT} \leq \mathrm{ULN})$ & $6.0(\mathrm{ALT} \leq \mathrm{ULN})$ \\
\hline & & & 8.0 (ALT 1-5× ULN) & 7.5 (ALT 1-5× ULN) \\
\hline Sensitivity, \% & 90.7 & 86.4 & 90.7 & 97.5 \\
\hline Specificity, \% & 37.5 & 38.5 & 53.8 & 41.3 \\
\hline PPV, \% & 62.2 & 61.4 & 69.0 & 65.3 \\
\hline NPV, \% & 78.0 & 71.4 & 83.6 & 93.5 \\
\hline $\mathrm{LR}+$ & 1.5 & 1.4 & 2.0 & 1.7 \\
\hline LR- & 0.2 & 0.4 & 0.2 & 0.1 \\
\hline No. of biopsy correctly avoided (\%) & 39 (37.5) & $40(38.4)$ & $56(53.8)$ & $43(41.3)$ \\
\hline No. of incorrect diagnosis (\%) & $11(10.5)$ & $16(15.3)$ & $11(9.3)$ & $3(2.8)$ \\
\hline
\end{tabular}


Table 3. Continued

\begin{tabular}{|c|c|c|c|c|}
\hline \multirow{2}{*}{ Variable } & \multicolumn{2}{|c|}{ ELF test } & \multicolumn{2}{|c|}{ LS value* } \\
\hline & Internal & External & Internal & External \\
\hline \multicolumn{5}{|l|}{ Confirmation strategy } \\
\hline \multicolumn{5}{|l|}{$\geq \mathrm{F} 3(\mathrm{n}=141)$} \\
\hline \multirow[t]{2}{*}{ Cutoff value } & 10.8 & 10.8 & $10.5($ ALT $\leq$ ULN $)$ & $9.0(\mathrm{ALT} \leq \mathrm{ULN})$ \\
\hline & & & 11.0 (ALT 1-5× ULN) & 12.0 (ALT 1-5× ULN) \\
\hline Sensitivity, \% & 24.8 & 24.8 & 63.2 & 66.0 \\
\hline Specificity, \% & 92.6 & 92.6 & 90.0 & 87.8 \\
\hline PPV, \% & 85.4 & 85.4 & 91.0 & 89.6 \\
\hline NPV, \% & 41.4 & 41.4 & 60.4 & 61.7 \\
\hline $\mathrm{LR}+$ & 3.4 & 3.4 & 6.3 & 5.4 \\
\hline LR- & 0.8 & 0.8 & 0.4 & 0.4 \\
\hline No. of biopsy correctly avoided (\%) & $35(24.8)$ & $35(24.8)$ & $91(64.5)$ & 95 (67.3) \\
\hline No. of incorrect diagnosis (\%) & $8(5.6)$ & $8(5.6)$ & $9(6.3)$ & $11(7.8)$ \\
\hline \multicolumn{5}{|l|}{$\mathrm{F} 4(\mathrm{n}=118)$} \\
\hline \multirow[t]{2}{*}{ Cutoff value } & 10.7 & 11.1 & $12.0(\mathrm{ALT} \leq \mathrm{ULN})$ & $12.0($ ALT $\leq$ ULN $)$ \\
\hline & & & 13.0 (ALT 1-5× ULN) & 12.0 (ALT $1-5 \times$ ULN) \\
\hline Sensitivity, \% & 28.8 & 21.2 & 59.2 & 60.0 \\
\hline Specificity, \% & 91.3 & 91.3 & 91.2 & 89.5 \\
\hline PPV, \% & 79.1 & 73.5 & 87.7 & 85.7 \\
\hline NPV, \% & 53.1 & 50.5 & 68.0 & 68.0 \\
\hline $\mathrm{LR}+$ & 3.3 & 2.8 & 6.7 & 5.7 \\
\hline LR- & 0.8 & 0.8 & 0.4 & 0.4 \\
\hline No. of biopsy correctly avoided (\%) & $34(28.8)$ & $25(21.1)$ & $71(60.1)$ & $72(61.0)$ \\
\hline No. of incorrect diagnosis (\%) & $9(7.6)$ & $9(7.6)$ & $10(8.4)$ & $12(10.1)$ \\
\hline
\end{tabular}

The accuracy of the LS-ELF algorithm using internal cutoff values is calculated from our study population. The external cutoff values describe the published results from the Wong's study. ${ }^{23}$

LS, liver stiffness; ELF, enhanced liver fibrosis; ALT, alanine aminotransferase; ULN, upper limit of normal; PPV, positive predictive value; NPV, negative predictive value; $\mathrm{LR}$, likelihood ratio.

${ }^{*} \mathrm{LS}$ values were expressed as kilopascals $(\mathrm{kPa})$.

nal and internal cutoffs are also summarized in Table 3. When ULN $_{\text {Korea }}$ was used, the overall results were similar (Supplementary Table 3).

\section{Diagnostic performance of the sequential and con- current LS-ELF algorithm to predict $\geq F 3$ and F4}

The diagnostic performances of the sequential LS-ELF algorithm and concurrent LS-ELF algorithms assessed using strategies by Castéra et al. ${ }^{10}$ and Boursier et $a{ }^{30}{ }^{30}$ to predict $\geq \mathrm{F} 3$ and F4 are shown in Table 4. When the sequential LS-ELF algorithm was applied using a confirmation and exclusion strategy, 69.4\% to $72.5 \%$ and $60.8 \%$ to $65.3 \%$ of patients could avoid LB to diagnose $\geq F 3$ and $F 4$, respectively, according to internal and external cutoff values. The proportion of patients who could avoid LB to diagnose $\geq F 3$ and F4, when the concurrent LS-ELF algorithm based on the strategies of Castéra and Boursier was applied, are also summarized in Table 4.

The proportion of patients with correctly avoided LB in predicting $\geq \mathrm{F} 3$ according to the sequential LS-ELF algorithm (69.4\% by internal cutoffs and $72.5 \%$ by external cutoffs) was significantly higher than the proportion of those with correctly avoided LB in predicting $\geq \mathrm{F} 3$ according to the concurrent LSELF algorithm by Castéra et al. ${ }^{10}$ (42.3\% using internal cutoffs and 59.0\% using external cutoffs) and Boursier et al. ${ }^{30}$ (57.7\%) (all $\mathrm{p}<0.05$ by McNemar test, with the exception of the borderline statistical significance between Boursier's strategy and the current study using external cutoff values $[p=0.057])$. Similarly, the proportion of patients with correctly avoided LB in predicting F4 was significantly higher with the sequential LSELF algorithm than the concurrent algorithms (all $\mathrm{p}<0.05$ by McNemar test except for the borderline statistical significance between Castéra's strategy and the current study using internal cutoff values $[\mathrm{p}=0.059])$. When $\mathrm{ULN}_{\mathrm{Korea}}$ was used, similar findings were observed (data not shown). 
Table 4. Diagnostic Performance of the Sequential and Concurrent LS-ELF Algorithms to Predict $\geq F 3$ and F4

\begin{tabular}{|c|c|c|c|c|c|}
\hline \multirow{3}{*}{ Variable } & \multicolumn{2}{|c|}{ Sequential combination } & \multicolumn{3}{|c|}{ Concurrent combination } \\
\hline & \multirow{2}{*}{ Internal cutoff } & \multirow{2}{*}{ External cutoff } & \multicolumn{2}{|c|}{ Using Castéra's strategy $^{10}$} & \multirow{2}{*}{$\begin{array}{l}\text { Using Boursier's } \\
\text { strategy }^{30}\end{array}$} \\
\hline & & & Internal cutoff & External cutoff & \\
\hline \multicolumn{6}{|l|}{$\geq \mathrm{F} 3$} \\
\hline Sensitivity, \% & 97.0 & 97.4 & 66.7 & 100.0 & 97.9 \\
\hline Specificity, \% & 67.8 & 40.2 & 90.1 & 55.6 & 88.9 \\
\hline PPV, \% & 81.5 & 63.6 & 92.2 & 79.7 & 93.9 \\
\hline NPV, \% & 93.8 & 93.5 & 60.8 & 100.0 & 96.0 \\
\hline $\mathrm{LR}+$ & 3.0 & 1.6 & 6.8 & 2.25 & 8.8 \\
\hline LR- & 0.0 & 0.1 & 0.4 & 0.00 & 0.0 \\
\hline No. of biopsy correctly avoided (\%) & $154(69.4)$ & $161(72.5)$ & $94(42.3)$ & $131(59.0)$ & $128(57.7)$ \\
\hline No. of incorrect diagnosis (\%) & $36(16.2)$ & $24(10.8)$ & $8(3.6)$ & $36(16.2)$ & $12(5.4)$ \\
\hline \multicolumn{6}{|l|}{$\mathrm{F} 4$} \\
\hline Sensitivity, $\%$ & 86.4 & 97.2 & 44.9 & 100.0 & 96.6 \\
\hline Specificity, $\%$ & 84.6 & 51.9 & 88.5 & 63.5 & 90.4 \\
\hline PPV, \% & 86.4 & 77.8 & 81.5 & 75.6 & 91.9 \\
\hline NPV, \% & 84.6 & 91.3 & 58.6 & 100.0 & 95.9 \\
\hline $\mathrm{LR}+$ & 5.6 & 2.0 & 3.9 & 2.7 & 10.0 \\
\hline LR- & 0.2 & 0.1 & 0.6 & 0.0 & 0.0 \\
\hline No. of biopsy correctly avoided (\%) & $145(65.3)$ & $135(60.8)$ & 53 (23.9) & $96(43.2)$ & $110(49.5)$ \\
\hline No. of incorrect diagnosis (\%) & $36(16.2)$ & $32(14.4)$ & $12(5.4)$ & $38(17.1)$ & $14(6.3)$ \\
\hline
\end{tabular}

LS, liver stiffness; ELF, enhanced liver fibrosis; PPV, positive predictive value; NPV, negative predictive value; LR, likelihood ratio.

\section{Schematic diagram of the sequential LS-ELF algorithm}

A schematic diagram of the sequential LS-ELF algorithm is shown in Fig. 2. Using the internal cutoff values, 83 patients were excluded from $\geq F$ 3 (58 with correct exclusion and 25 with incorrect exclusion). In addition, 107 patients were confirmed to have $\geq$ F3 (96 with correct confirmation and 11 with incorrect confirmation) (Fig. 2A). Regarding an F4 diagnosis, 87 patients were excluded (59 with correct exclusion and 28 with incorrect exclusion). Furthermore, among the 94 patients confirmed with F4, 86 had a correct diagnosis and eight had an incorrect diagnosis (Fig. 2B). Diagrams of diagnoses using external cutoff values to predict $\geq F$ 3 and F4 are shown in Fig. $2 \mathrm{C}$ and D. In addition, all of the diagnostic diagrams using $\mathrm{ULN}_{\text {Korea }}$ showed similar patterns in terms of predicting $\geq \mathrm{F} 3$ and F4 (Supplementary Fig. 2).

\section{Prognostic value of LS-ELF algorithm}

When the study population was divided into two groups according to the combined LS-ELF algorithm using the internal cutoff values for prediction of $\geq \mathrm{F} 3$, a significantly higher incidence of HCC was observed in patients with advanced fibrosis confirmed $(n=107)$ than in patients with advanced fibrosis excluded ( $n=83)(p<0.001$ by log-rank test) (Supplementary Fig. 3). When the internal cutoff value for prediction of F4 and external cutoff values for prediction of $\geq \mathrm{F} 3$ and $\mathrm{F} 4$ were used, similar findings were also observed (data not shown).

\section{DISCUSSION}

Although TE can accurately assess the degree of liver fibrosis, its diagnostic performance for detecting early stage liver fibrosis is unsatisfactory. ${ }^{19}$ Similarly, ELF can reliably predict the degree of liver fibrosis. ${ }^{15-18}$ Recently, Wong et al. ${ }^{23}$ proposed a sequential diagnostic algorithm using LS and ELF values, which was effective for avoiding LB in CHB patients. In our study, we found that the LS-ELF algorithm prevented unnecessary LB in $69.4 \%$ to $72.5 \%$ of patients with advanced liver fibrosis, similar to the results of a Hong Kong study (61\% to 66\%). Moreover, the sequential LS-ELF algorithm was significantly superior to the concurrent use of two surrogates in terms of avoiding LB in CHB patients.

This study had several clinical strengths. First, we validated the diagnostic performance of a recently proposed LS-ELF algorithm. The diagnostic accuracy of LS value for predicting liver fibrosis was similar in our cohort and the Hong Kong study ${ }^{23}$ (AUC, 0.857 to 0.887 vs 0.82 to 0.83 ), whereas the accuracy of the ELF test in our cohort was higher than that in the Hong Kong study ${ }^{23}$ (AUC, 0.703 to 0.802 vs 0.59 to 0.69). Nevertheless, our data demonstrated that the combined use of LS and 
A

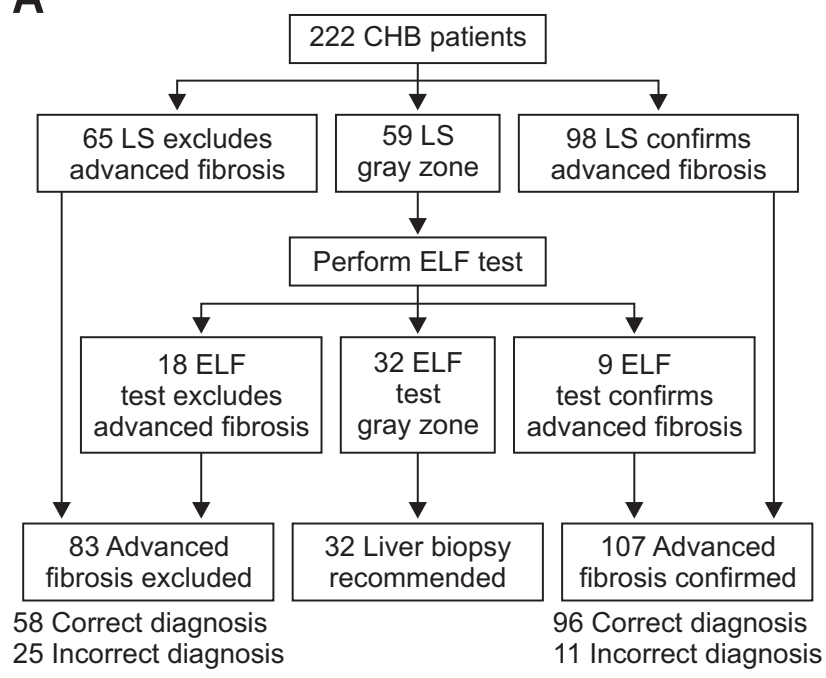

C

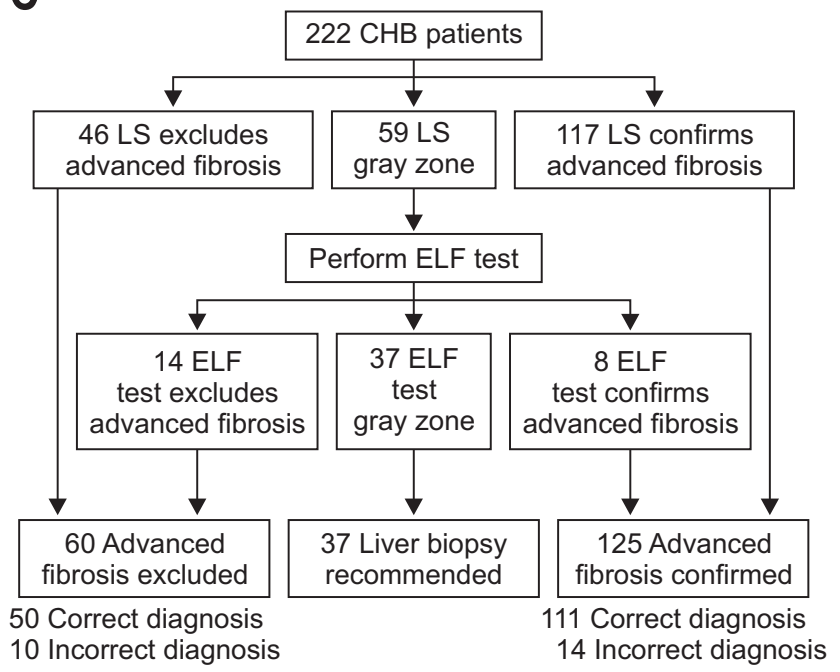

B
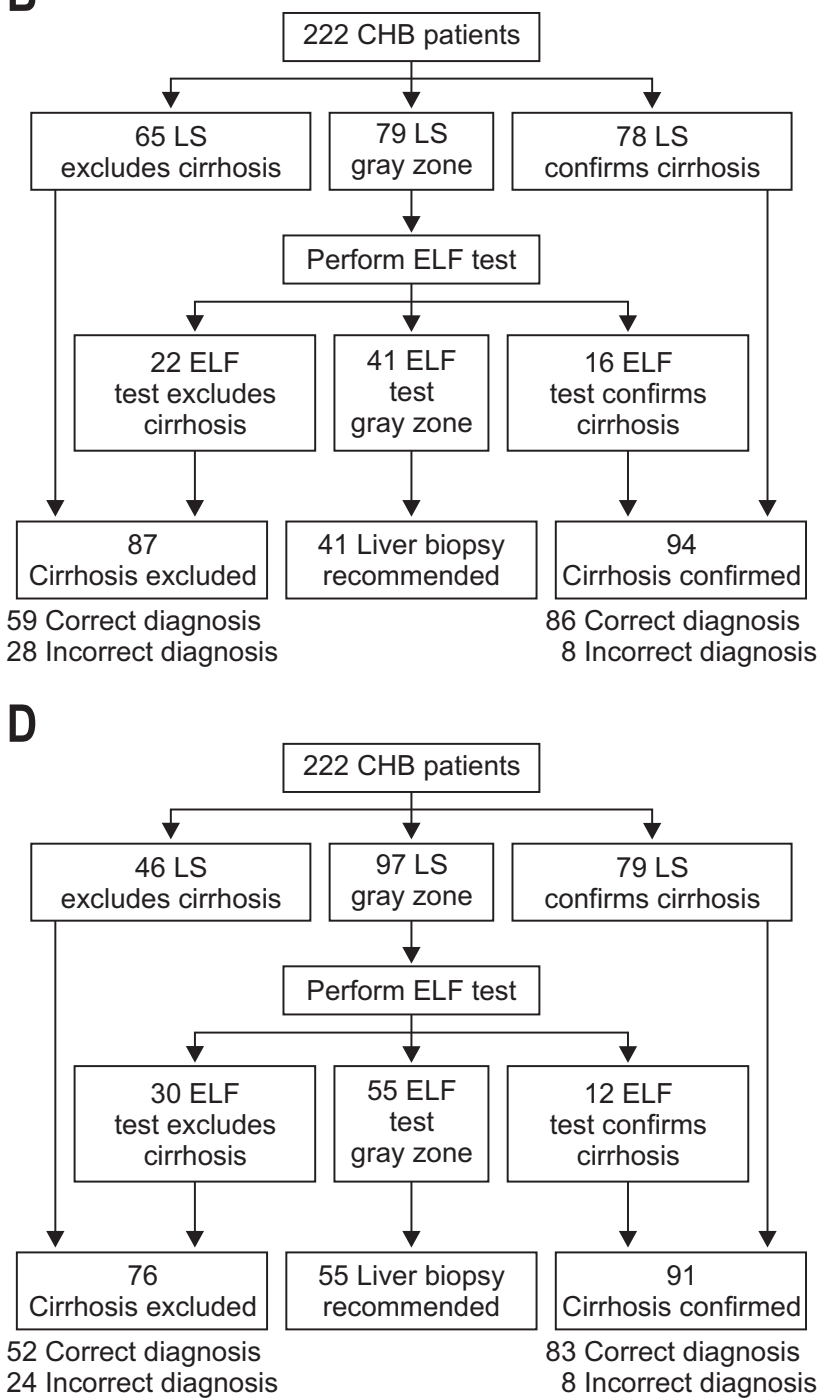

Fig. 2. Prediction of $\geq F 3$ and F4 stages using the combined LS-ELF algorithm with internal (A, B) and external cutoff values (C, D). LS, liver stiffness; ELF, enhanced liver fibrosis; CHB, chronic hepatitis B.

ELF values has improved diagnostic accuracy, which is consistent with Wong et al..$^{23}$ Because ELF shows better performance in predicting early stage fibrosis ( $\geq \mathrm{F} 2)$ (AUC: 0.802 in our study and 0.82 to 0.90 in previous studies, ${ }^{16-18}$ ) and LS shows better performance in predicting advanced-stage fibrosis $(\geq F$ ) (AUC: 0.887 in our study and 0.83 to 0.90 in previous studies, ${ }^{23,32}$ ) their combined use enhances the overall accuracy of assessment of fibrotic burden. Similarly, the combined use of two surrogates, such as the combination of FT and LS by Castéra et al..$^{10}$ in CHC patients and the combination of FibroMeter and LS values by Boursier et al., ${ }^{30}$ improves diagnostic accuracy compared to use of either marker alone, which supports our findings. In addition, we found the prognostic value of LS-ELF algorithm by showing the significantly higher incidence rate of HCC in patients with advanced fibrosis or cirrhosis confirmed than in patients with advanced fibrosis or cirrhosis excluded. Although validation studies in other ethnic groups are required, the LS-ELF algorithm can be applied to CHB patients in Asian counties, which have a high prevalence of HBV.

Second, our study revealed that the sequential use of LS and ELF values is superior to their concurrent use. According to the sequential LS-ELF algorithm, the proportion of patients with correctly avoided LB in predicting $\geq \mathrm{F} 3(69.4 \%$ to $72.5 \%$ vs $42.3 \%$ to $59.0 \%)$ and F4 $(60.8 \%$ to $65.3 \%$ vs $23.9 \%$ to $49.5 \%)$ was significantly higher than that of concurrent use of LS and ELF. Although further prospective studies confirming our results and dealing with cost-efficiency of this diagnostic algorithm are required, sequential strategy may potentially lead to cost savings. That is, the concurrent use of LS value and the ELF test to screen all of the CHB patients incurs expenses compared to the sequential measurement of two surrogates.

Third, we validated the LS-ELF algorithm in various clinical 
settings to investigate the potential influence of clinical confounders. To reveal whether the main results can be influenced according to the specific characteristics of our cohort, we tested the LS-ELF algorithm according to both internal and external cutoff values from Hong $\mathrm{Kong}^{23}$ and the different ULN of the ALT level between Hong Kong (67 IU/L for males and 55 IU/ L for females) and Korea (40 IU/L for both sexes). The overall results were not influenced by several potential confounders, which supports the applicability of the LS-ELF algorithm. However, due to the different distribution of fibrosis stage and ALT level between our work and the Hong Kong study, further research is required.

In our study, the internal cutoff values of LS according to fibrosis stage were 9.0 to $11.0 \mathrm{kPa}$ and those of ELF were 8.4 to 9.8, similar to previous reports. ${ }^{23,25,28,29}$ Although the LS cutoff increased with increasing ALT level, LS values were not correlated with ALT levels ( $p=0.067)$; therefore, ALT only had minimal effects on the internal cutoff values of LS and ELF in our study, in contrast to previous reports. ${ }^{31,33-35}$ This might be due to attenuation of the influence of a high ALT level by exclusion of CHB patients with ALT levels $>5 \times$ ULN. The LS results of CHB patients with acute exacerbation are not reliable for diagnosing cirrhosis, ${ }^{34}$ and the cutoff LS values of patients with ALT levels 1-5x ULN were higher than those of patients with normal ALT levels. ${ }^{31,33}$ However, a recent multicenter study reported that the overestimating influence of an ALT 1-10x ULN was modest, as the accuracy of LS values was similar irrespective of ALT adjustment. ${ }^{35}$ Due to this controversy, we stratified the patients according to ALT level (ALT $\leq$ ULN vs ALT 1-5x ULN) and ULN criteria (ULN vs ULN $_{\text {Korea }}$ ), but ALT level had no significant effects on the results. In this study, ELF values did not correlate with ALT levels, and thus different ELF cutoff values according to ALT level could not be used.

We are aware of several issues with this study. First, because of its retrospective design, the study was subject to potential selection bias. Histological information for all of the patients who started antiviral therapy during the study period could not be obtained, despite confirming the diagnostic accuracy of the LS-ELF algorithm. Thus, further prospective validation of the LS-ELF algorithm is required before it can be applied in clinical practice. In addition, although we excluded patients with the length of liver samples $<1.5 \mathrm{~cm}$ not only for securing more reliable pathological interpretation, but also for obtaining a large sample size as much as we could, the relatively insufficient quality of liver sample might have limited the accuracy of histological assessment of liver fibrosis. Second, because validation of the LS-ELF algorithm in patients with ALT<5×ULN was restricted to prevent the confounding influence of high ALT levels on LS values, it cannot be recommended for patients with AT values $>5 \times$ ULN. However, most of the unusually high LS during acute liver injury showed a progressive and rapid decrease in parallel with a decrease in ALT levels. ${ }^{34}$ Thus, applicability of the
LS-ELF algorithm after stabilizing ALT level by antiviral or conservative therapy should be investigated. Third, probably due to low NPV of exclusion strategy, our study showed higher rates of incorrect diagnosis of patients who avoided LB compared to Wong et al. $^{23}$ (NPV, 69.9\% to $83.1 \%$ in this work vs $90 \%$ in Wong's study). Fourth, this study had a potential spectrum bias, with a high proportion of patients with cirrhosis (53.2\%). In contrast, in Wong et al., ${ }^{23}$ 15\% of patients had F3 fibrosis and 25\% had cirrhosis. However, despite the different fibrosis stage distribution between Korea and Hong Kong, the LS-ELF algorithm consistently performed better than either LS or ELF alone. Lastly, although the sequential LS-ELF diagnostic algorithm exhibited enhanced overall diagnostic accuracy, it is associated with higher costs than LS alone. Thus, a cost-efficiency analysis of this algorithm is warranted.

In conclusion, the sequential LS-ELF algorithm had a higher probability of preventing LB in CHB patients to diagnose advanced fibrosis and cirrhosis and performed significantly better than that of the concurrent algorithms. However, further prospective validation studies are required before this algorithm can be used clinically.

\section{CONFLICTS OF INTEREST}

No potential conflict of interest relevant to this article was reported.

\section{ACKNOWLEDGEMENTS}

This study was supported in part by the Basic Science Research Program through the National Research Foundation of Korea funded by the Ministry of Science, ICT \& Future Planning (2016R1A1A1A05005138).

\section{REFERENCES}

1. World Health Organization. Guidelines for the prevention, care and treatment of persons with chronic hepatitis B infection. Geneva: World Health Organization, 2015.

2. Yu SJ. A concise review of updated guidelines regarding the management of hepatocellular carcinoma around the world: 20102016. Clin Mol Hepatol 2016;22:7-17.

3. Lai CL, Yuen MF. Prevention of hepatitis B virus-related hepatocellular carcinoma with antiviral therapy. Hepatology 2013;57:399408.

4. Bolondi L, Sofia S, Siringo S, et al. Surveillance programme of cirrhotic patients for early diagnosis and treatment of hepatocellular carcinoma: a cost effectiveness analysis. Gut 2001;48:251-259.

5. Varbobitis I, Papatheodoridis GV. The assessment of hepatocellular carcinoma risk in patients with chronic hepatitis B under antiviral therapy. Clin Mol Hepatol 2016;22:319-326.

6. Rockey DC, Caldwell SH, Goodman ZD, Nelson RC, Smith AD; 
American Association for the Study of Liver Diseases. Liver biopsy. Hepatology 2009;49:1017-1044.

7. The French METAVIR Cooperative Study Group. Intraobserver and interobserver variations in liver biopsy interpretation in patients with chronic hepatitis C. Hepatology 1994;20(1 Pt 1):15-20.

8. Park SH, Kim SY, Suh CH, et al. What we need to know when performing and interpreting US elastography. Clin Mol Hepatol 2016;22:406-414.

9. Bravo AA, Sheth SG, Chopra S. Liver biopsy. N Engl J Med 2001;344:495-500.

10. Castéra L, Vergniol J, Foucher J, et al. Prospective comparison of transient elastography, FibroTest, APRI, and liver biopsy for the assessment of fibrosis in chronic hepatitis C. Gastroenterology 2005;128:343-350.

11. Heo JY, Kim SU, Kim BK, et al. Use of Wisteria floribunda agglutinin-positive human Mac-2 binding protein in assessing risk of hepatocellular carcinoma due to hepatitis B virus. Medicine (Baltimore) 2016;95:e3328.

12. Castera L. Hepatitis B: are non-invasive markers of liver fibrosis reliable? Liver Int 2014;34 Suppl 1:91-96.

13. Wong GL, Espinosa WZ, Wong VW. Personalized management of cirrhosis by non-invasive tests of liver fibrosis. Clin Mol Hepatol 2015;21:200-211.

14. Kim BK, Kim HS, Yoo EJ, et al. Risk assessment of clinical outcomes in Asian patients with chronic hepatitis B using enhanced liver fibrosis test. Hepatology 2014;60:1911-1919.

15. Fagan KJ, Pretorius CJ, Horsfall LU, et al. ELF score $\geq 9.8$ indicates advanced hepatic fibrosis and is influenced by age, steatosis and histological activity. Liver Int 2015;35:1673-1681.

16. Irvine KM, Wockner LF, Shanker M, et al. The enhanced liver fibrosis score is associated with clinical outcomes and disease progression in patients with chronic liver disease. Liver Int 2016;36:370-377.

17. Guéchot J, Trocmé C, Renversez JC, Sturm N, Zarski JP; ANRS HC EP 23 Fibrostar Study Group. Independent validation of the enhanced liver fibrosis (ELF) score in the ANRS HC EP 23 Fibrostar cohort of patients with chronic hepatitis C. Clin Chem Lab Med 2012;50:693-699.

18. Kim BK, Kim HS, Park JY, et al. Prospective validation of ELF test in comparison with Fibroscan and FibroTest to predict liver fibrosis in Asian subjects with chronic hepatitis B. PLoS One 2012;7:e41964.

19. Seo YS, Kim MY, Kim SU, et al. Accuracy of transient elastography in assessing liver fibrosis in chronic viral hepatitis: a multicentre, retrospective study. Liver Int 2015;35:2246-2255.

20. Kim DY, Song KJ, Kim SU, et al. Transient elastography-based risk estimation of hepatitis B virus-related occurrence of hepatocellular carcinoma: development and validation of a predictive model. Onco Targets Ther 2013;6:1463-1469.

21. Lee HW, Yoo EJ, Kim BK, et al. Prediction of development of liver- related events by transient elastography in hepatitis B patients with complete virological response on antiviral therapy. Am J Gastroenterol 2014;109:1241-1249.

22. Wong GL, Chan HL, Wong CK, et al. Liver stiffness-based optimization of hepatocellular carcinoma risk score in patients with chronic hepatitis B. J Hepatol 2014;60:339-345.

23. Wong GL, Chan HL, Choi PC, et al. Non-invasive algorithm of enhanced liver fibrosis and liver stiffness measurement with transient elastography for advanced liver fibrosis in chronic hepatitis B. Aliment Pharmacol Ther 2014;39:197-208.

24. Korean Liver Cancer Study Group (KLCSG); National Cancer Center, Korea (NCC). 2014 Korean Liver Cancer Study Group-National Cancer Center Korea practice guideline for the management of hepatocellular carcinoma. Korean J Radiol 2015;16:465-522.

25. Korean Association for the Study of the Liver. KASL clinical practice guidelines: management of chronic hepatitis B. Clin Mol Hepatol 2016;22:18-75.

26. Batts KP, Ludwig J. Chronic hepatitis: an update on terminology and reporting. Am J Surg Pathol 1995;19:1409-1417.

27. Kim JH, Kim MN, Han KH, Kim SU. Clinical application of transient elastography in patients with chronic viral hepatitis receiving antiviral treatment. Liver Int 2015;35:1103-1115.

28. Kim MN, Kim SU, Kim BK, et al. Increased risk of hepatocellular carcinoma in chronic hepatitis B patients with transient elastography-defined subclinical cirrhosis. Hepatology 2015;61:1851-1859.

29. Park MS, Kim SU, Kim BK, et al. Prognostic value of the combined use of transient elastography and FibroTest in patients with chronic hepatitis B. Liver Int 2015;35:455-462.

30. Boursier J, Vergniol J, Sawadogo A, et al. The combination of a blood test and Fibroscan improves the non-invasive diagnosis of liver fibrosis. Liver Int 2009;29:1507-1515.

31. Chan HL, Wong GL, Choi PC, et al. Alanine aminotransferasebased algorithms of liver stiffness measurement by transient elastography (Fibroscan) for liver fibrosis in chronic hepatitis B. J Viral Hepat 2009;16:36-44.

32. Trembling PM, Lampertico P, Parkes J, et al. Performance of enhanced liver fibrosis test and comparison with transient elastography in the identification of liver fibrosis in patients with chronic hepatitis B infection. J Viral Hepat 2014;21:430-438.

33. Fung J, Lai CL, Cheng C, Wu R, Wong DK, Yuen MF. Mild-tomoderate elevation of alanine aminotransferase increases liver stiffness measurement by transient elastography in patients with chronic hepatitis B. Am J Gastroenterol 2011;106:492-496.

34. Wong GL, Wong VW, Choi PC, et al. Increased liver stiffness measurement by transient elastography in severe acute exacerbation of chronic hepatitis B. J Gastroenterol Hepatol 2009;24:10021007.

35. Wong VW, Lampertico P, de Lédinghen V, et al. Probability-based interpretation of liver stiffness measurement in untreated chronic hepatitis B patients. Dig Dis Sci 2015;60:1448-1456. 